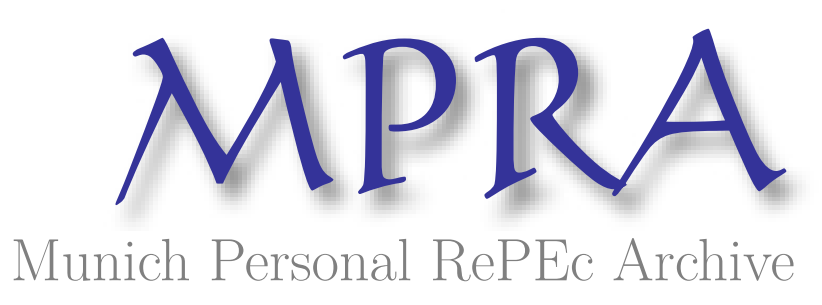

\title{
Bonds futures: Delta? No gamma!
}

Henrard, Marc

BIS

12 April 2006

Online at https://mpra.ub.uni-muenchen.de/2249/

MPRA Paper No. 2249, posted 14 Mar 2007 UTC 


\title{
BONDS FUTURES: DELTA? NO GAMMA!
}

\author{
MARC HENRARD
}

\begin{abstract}
Bond futures are liquid but complex instruments. Here they are analysed in a onefactor Gaussian HJM model. The in-the-model delta and out-of-the-model delta and gamma are studied. An explicit formula is provided for in-the-model delta. The out-of-the-model delta and gamma are equivalent to partial derivatives with respect ot discount factors. In particular cases the derivative can not be obtain by standard techniques. The same situations lead to cases where the gammas (second order partial derivatives) do not exists.

Copyright (c) 2006 by Marc Henrard.
\end{abstract}

\section{INTRODUCTION}

Bond futures are very liquid instruments and are often consider as vanilla products, after all they are just futures. Behind this simplifying name a lot of complexity is hidden. One of them is the delivery option: the short side of the futures can deliver any bonds in a pre-defined basket. This embedded option creates a real complexity in the pricing and risk management of the product. Bond futures are exotic products.

With the EUR very long term rates close to $4 \%$, the BUXL futures is almost at-the-money. The USD bond and notes rates have crossed the $5 \%$ level and their futures may also be soon at-the-money. It is probably the good moment to ensure that the delivery option embedded in the bond futures is well understood and managed.

This note should be read as a sequel to the article Henrard (2006a). A semi-explicit valuation formula for the delivery option in the Gaussian HJM one-factor model was proposed. Beyond the price of the instrument is their hedging. In a model with deterministic volatility, the theoretical hedging is done only with the underlyings. In term structure models the underlyings are (zerocoupon) bonds. As the model used here is one-factor any bond can be used to hedge any option. This hedging with the underlying is called delta hedging or, to emphasize the fact that the hedging is coherent with the model, in-the-model delta hedging. This risk measure is the only one theoritically justified.

There are other measures of rate or price dependency used in practice: the out-of-the-model delta and gamma. They correspond to the first and second order derivatives of the price with respect to the (zero-coupon) bond prices (or rates). Those figure are used in practice and easy to compute (approximativelly) by symmetrical difference. Neverthless those figure have no theoretical justification and even their existence is not garanted.

The in-the-model delta is computed in the main theorem of the note. The formula is obtained in two different ways. The first one through partial derivatives computations. This is a standard approach for example used in (Lamberton and Lapeyre, 2000, Section 4.3.3) for Black-Sholes formula or in Henrard (2003) for the swaptions in the same model that is used here. In that proof one encounters some technical difficulties. It is not clear that the partial derivatives always exist. Generically they do but in some singular cases some part of the formula is not differentiable (infinite slope). The fact that some part of a composition is not differentiable does not prove that the total composition is non-differentiable but creates a significant technical difficulty. The theorem is proved when the technical difficulty does not appear.

Date: First version: 12 April 2006; this version: 1 May 2006.

Key words and phrases. Bond future, delivery option, delta, gamma, HJM gaussian model, in-the-model, outof-the-model.

JEL classification: G13, E43.

AMS mathematics subject classification: 91B28, 91B24, 91B70. 
A second proof using the structure of the model and no partial derivatives is provided. The proof is valid for all cases. The proof is using heavily the structure of the problem created by the model. It does not require technical conditions to be valid but require higher level view of the valuation formula and of the structure of the problem.

With the second proof the theoretical approach is cleaned but not the practical one. It is still not clear if the partial derivatives always exists. The definitive answer is not provided here. Nevertheless through the example of the last section it is shown that the technical condition of the first proof is not only technical but results from difficulties in the problem it-self. It is shown that in a specific example the second order partial derivative with respect to one of the prices does not exist. The example is quite simple as it involves only two bonds in the delivery basket with only one cash-flow each.

The conclusion of the example is that the gamma (which is always out-of-the-model) is not always well defined. It can tend to infinity in some points (even away from the expiry date) and can be discountinuous. This can be the start of thoughs on the usage of gamma as a risk measure and an indicator for limits.

\section{Futures AND HJM MOdeL}

The notation and description are the same as in Henrard (2006a).

Suppose there are $N$ bonds in the basket. Each of them $(1 \leq i \leq N)$ has $n_{i}$ coupons after the delivery date $t_{0}$; the cash flows amount are $c_{i, j}$ and are paid in $t_{i, j}$. Let $A_{i}$ denote the accrued interests at delivery and $K_{i}$ the convertion factor. The fixing take place in $\theta \leq t_{0}$. The price in $t$ of a zero coupon bond with maturity $u$ is denoted $P(t, u)$. The time $t$ futures price is denoted by $F_{t}$

The futures price is

$$
F_{\theta}=\min _{1 \leq i \leq N}\left(\sum_{j=1}^{n_{i}} \frac{c_{i, j}}{K_{i}} \frac{P\left(\theta, t_{i, j}\right)}{P\left(\theta, t_{0}\right)}-\frac{A_{i}}{K_{i}}\right) .
$$

The valuation of the futures in this framework is done using the Gaussian HJM one-factor model. Preliminary results on that model can be found in the above paper.

When the discount curve $P(t,$.$) is absolutely continuous, which is something that is always the$ case in practice as the curve is constructed by some kind of interpolation, there exists $f(t, u)$ such that

$$
P(t, u)=\exp \left(-\int_{t}^{u} f(t, s) d s\right) .
$$

The idea of Heath et al. (1992) was to exploit this property by modeling $f$ with a stochastic differential equation

$$
d f(t, u)=\mu(t, u) d t+\sigma(t, u) d W_{t}
$$

for some suitable (stochastic) $\mu$ and $\sigma$ and deducing the behavior of $P$ from there. To ensure the arbitrage-free property of the model, a relationship between the drift and the volatility is required. Here the volatility, the drift, the rate and the Brownian motion are 1-dimensional. The model technical details can be found in the original paper or in the chapter Dynamical term structure model of Hunt and Kennedy (2004). The notation of the later is used.

The probability space is $\left(\Omega,\left\{\mathcal{F}_{t}\right\}, \mathcal{F}, \mathbb{P}\right)$. The filtration $\mathcal{F}_{t}$ is the (augmented) filtration of the standard Brownian motion $\left(W_{t}\right)_{0 \leq t \leq T}$. To simplify the writing in the rest of the paper, the bond volatility is denoted

$$
\nu(t, u)=\int_{t}^{u} \sigma(t, s) d s .
$$

Let $N_{t}=\exp \left(\int_{0}^{t} r_{s} d s\right)$ be the cash-account numeraire with $\left(r_{s}\right)_{0 \leq s \leq T}$ the short rate given by $r_{t}=f(t, t)$. The equations of the model in the numeraire measure $\mathbb{N}$ associated to $N_{t}$ are

$$
d f(t, u)=\sigma(t, u) \nu(t, u) d t+\sigma(t, u) d W_{t}
$$


or

$$
d P^{N}(t, u)=-P^{N}(t, u) \nu(t, u) d W_{t}
$$

The notation $P^{N}(t, s)$ designates the numeraire rebased value of $P$, i.e. $P^{N}(t, s)=N_{t}^{-1} P(t, s)$.

Like in the case of swaption analysed in Henrard (2006b), a separability condition is used to obtain explicit results. The condition is similar to the condition to have Markovian short rate obtained by Carverhill (1994).

H: The function $\sigma$ satisfies $\sigma(t, u)=g(t) h(u)$ for some positive function $g$ and $h$.

The condition is satisfied by the extended Vasicek model.

The two following technical lemmas were presented in Henrard (2006b) for the Gaussian onefactor HJM. Similar formulas can be found in (Brody and Hughston, 2004, (3.3),(3.4)) in the framework of coherent interest-rate models and in Nunes and de Oliveira (2004) for multi-factor Gaussian HJM.

Lemma 1. Let $0 \leq t \leq u \leq v$. In HJM framework the price of the zero coupon bond is

$$
P(u, v)=\frac{P(t, v)}{P(t, u)} \exp \left(-\int_{t}^{u}(\nu(s, v)-\nu(s, u)) d W_{s}-\frac{1}{2} \int_{t}^{u}\left(\nu(s, v)^{2}-\nu(s, u)^{2}\right) d s\right) .
$$

The discount factor ratio in the futures price can be written as

$$
\frac{P\left(\theta, t_{i, j}\right)}{P\left(\theta, t_{0}\right)}=\frac{P\left(t, t_{i, j}\right)}{P\left(t, t_{0}\right)} \beta_{i, j} \exp \left(-\alpha_{i, j}^{2}-\alpha_{i, j} X\right)
$$

with

$$
\begin{gathered}
\beta_{i, j}=\beta_{i, j}(t, \theta)=\exp \left(-\int_{t}^{\theta} \nu\left(s, t_{0}\right)\left(\nu\left(s, t_{i, j}\right)-\nu\left(s, t_{0}\right)\right) d s\right), \\
\alpha_{i, j}^{2}=\alpha_{i, j}^{2}(t, \theta)=\int_{t}^{\theta}\left(\nu\left(s, t_{i, j}\right)-\nu\left(s, t_{0}\right)\right)^{2} d s
\end{gathered}
$$

and $X$ a $\mathbb{N}$-standard normal random variable. The separability condition $(\mathrm{H})$ is used to prove that the same variable $\mathrm{X}$ can be used for all bonds and coupons.

Using the notation

$$
d_{i, j}=d_{i, j}(t, \theta)=\frac{c_{i, j}}{K_{i}} \beta_{i, j},
$$

$e_{i}=A_{i} / K_{i}, t_{i, 0}=t_{0}$ and $d_{i, 0}(t, \theta)=-e_{i}$ the future price is

$$
F_{\theta}=\min _{1 \leq i \leq N}\left(\sum_{j=0}^{n_{i}} d_{i, j}(t, \theta) \frac{P\left(t, t_{i, j}\right)}{P\left(t, t_{0}\right)} \exp \left(-\frac{1}{2} \alpha_{i, j}^{2}(t, \theta)-\alpha_{i, j}(t, \theta) X\right)\right) .
$$

The functions in the minimum are denoted

$$
\left.f_{i}(x)=f_{i}(x ; t, \theta)\right)=\sum_{j=0}^{n_{i}} d_{i, j} \frac{P\left(t, t_{i, j}\right)}{P\left(t, t_{0}\right)} \exp \left(-\frac{1}{2} \alpha_{i, j}^{2}-\alpha_{i, j} x\right) .
$$

\section{Delta}

In bond futures the value that should be attained is not the futures price but its gain process. The futures price is only a screen number. It drives the marginning process but the price is not a pay-off. The pay-off that should be attained in the hedging process is the gain associated to the futures denoted $G_{t}(F, N)$ (see (Hunt and Kennedy, 2004, Chapter 12)). The numeraire used here is the cash account (denoted $\zeta^{-1}$ in Hunt and Kennedy (2004)). With that numeraire the discounted gain process satisfies (Hunt and Kennedy, 2004, Equation 12.6)

If the process is written as

$$
G_{t}(F, N)=\int_{0}^{t} N_{u}^{-1} d F_{u} .
$$

$$
G_{t}=\int_{0}^{t} \phi_{u} d B_{u}^{N}
$$


for some asset $B_{u}$, then the random process $\phi$ is a hedging strategy. To obtain this the futures process is written as

$$
d F_{t}=N_{t} \phi_{t} d B_{t}^{N} .
$$

The hedging bond is described below and the hedging strategy is described in the following theorem.

Let $B$ be a bond with cash-flow dates $s_{i}(1 \leq i \leq l)$ and cash-flow amounts $b_{i}$. The discounting value of the bond $B_{t}^{N}$ satisfy the equation

$$
d B_{t}^{N}=-\sum_{i=1}^{l} b_{i} P^{N}\left(t, s_{i}\right) \nu\left(t, s_{i}\right) d W_{t} .
$$

Theorem 1. In the separable one-factor gaussian HJM model, the hedging strategy in $t$ for the bond futures is to hold the quantity

$\Delta_{t}=\frac{1}{P\left(t, t_{0}\right)} \frac{\sum_{i=1}^{k} \sum_{j=0}^{n_{m_{i}}} d_{m_{i}, j} P\left(t, t_{m_{i}, j}\right)\left(N\left(\kappa_{i}+\alpha_{m_{i}, j}\right)-N\left(\kappa_{i-1}+\alpha_{m_{i}, j}\right)\right) \nu\left(t, t_{m_{i}, j}\right)-F_{t} P\left(t, t_{0}\right) \nu\left(t, t_{0}\right)}{\sum_{i=1}^{l} b_{i} P\left(t, s_{i}\right) \nu\left(t, s_{i}\right)}$

of the bond $B$.

Proof (Using partial derivatives). Let $x=P(t)=\left(P\left(t, t_{0}\right), \ldots, P\left(t, t_{m_{i}, j}\right), \ldots\right)=\left(\ldots, P_{(i, j)}, \ldots\right)=$ $P$. The order in which the bonds $P_{(i, j)}\left(1 \leq i \leq k, 1 \leq j \leq n_{m_{i}}\right)$ are sorted in th vector is irrelevant for the argument that follows. The value of the bond is

$$
F_{t}=F(t, P, \kappa) .
$$

$f_{i}(x, P)=\sum_{j=0}^{n_{m_{i}}} d_{m_{i}, j} P_{(i, j)} \exp \left(-\frac{1}{2} \alpha_{m_{i}, j}^{2}-\alpha_{m_{i}, j} x\right)-\sum_{j=0}^{n_{m_{i+1}}} d_{m_{i+1}, j} P_{(i+1, j)} \exp \left(-\frac{1}{2} \alpha_{m_{i+1}, j}^{2}-\alpha_{m_{i+1}, j} x\right)$.

The dependency of $F$ on $P$ appears directly and indirectly through $\kappa$. It is first proved that

$$
\frac{d}{d P_{(i, j)}} F(t, P, \kappa(P))=\frac{\partial}{\partial P_{(i, j)}} F(t, P, \kappa) .
$$

The (total) derivative of $F$ with respect to $P\left(t, t_{0}\right)$ is denote $D_{(0)} F$ and the one with respect to $P_{(i, j)}$ by $D_{(i, j)} F$.

Generically the quantity $D_{1} f \neq 0$ and one can use the implicit function theorem to obtain the existence and derivability of $\kappa$ as a function of $P$. One can then compute $D_{(i, j)} F$ by composition rule.

The result is proved for $p>0$, the other case is similar. Using the explicit formula for the future price (Henrard, 2006a, Equation 2)

$$
\begin{aligned}
& D_{(p, q)} F=\frac{1}{P\left(t, t_{0}\right)}\left(d_{m_{p}, q} N\left(\kappa_{p}+\alpha_{m_{p}, q}\right)-d_{m_{p}, q} N\left(\kappa_{p-1}+\alpha_{m_{p}, q}\right)\right) \\
& +\sum_{i=1}^{k-1} \sum_{j=0}^{n_{m_{i}}} d_{m_{i}, j} P_{(i, j)} N^{\prime}\left(\kappa_{i}+\alpha_{m_{i}, j}\right) D_{(p, q)} \kappa_{i} \\
& -\sum_{i=1}^{k-1} \sum_{j=0}^{n_{m_{i+1}}} d_{m_{i+1}, j} P_{(i+1, j)} N^{\prime}\left(\kappa_{i}+\alpha_{m_{i+1}, j}\right) D_{(p, q)} \kappa_{i}
\end{aligned}
$$

The normal density can be split in

$$
N^{\prime}\left(\kappa_{i}+\alpha_{m_{i}, j}\right)=\exp \left(-\frac{1}{2} \alpha_{m_{i}, j}^{2}-\alpha_{m_{i}, j} \kappa_{i}\right) \frac{1}{\sqrt{2 \pi}} \exp \left(-\frac{1}{2} \kappa_{i}^{2}\right)
$$

By using that split, grouping the factors of $D_{(p, q)} \kappa_{i}$ and the using Equation 2 one proves that only the first line of Equation 3 is non null. 
The derivative is given by

$$
D_{(p, q)} F=\frac{1}{P\left(t, t_{0}\right)} d_{m_{p}, q}\left(N\left(\kappa_{p}+\alpha_{m_{p}, q}\right)-N\left(\kappa_{p-1}+\alpha_{m_{p}, q}\right)\right)
$$

and

$$
D_{(0)} F=-\frac{1}{P^{2}\left(t, t_{0}\right)} \sum_{i=1}^{k} \sum_{j=1}^{n_{m_{i}}} d_{m_{i}, j} P_{(i, j)}\left(N\left(\kappa_{i}+\alpha_{m_{i}, j}\right)-N\left(\kappa_{i-1}+\alpha_{m_{i}, j}\right)\right)=-\frac{1}{P\left(t, t_{0}\right)} F_{t} .
$$

Like in Henrard (2003) the hedging is given by

$$
\phi_{t}=\frac{\sum_{i=1}^{k} \sum_{j=0}^{n_{m_{i}}} P_{(i, j)} \nu\left(t, t_{m_{i}, j}\right) D_{(i, j)} F+P_{(0)} \nu\left(t, t_{0}\right) D_{(0)} F}{\sum_{i=1}^{l} P\left(t, s_{i}\right) \nu\left(t, s_{i}\right) b_{i}}
$$

The result follows using the description of $D_{(i, j)} F$ and $D_{(0)} F$ given above.

There exists examples where the non-degenaracity condition $D_{1} f_{i}\left(\kappa_{i}, P\right) \neq 0$ is not satisfied. In those conditions the proof given does not hold anymore. Also in this context a small perturbation of the initial conditions $P$ can modify (substantially) the number of crossing.

The non-robustness too small perturbation is not in contradition with the strong robustness result proved in Henrard (2006a) for all movements admissible in the gaussian HJM model. Here the initial condition are perturbed in a way non compatible with the model. The individual derivatives computed $D_{(p, q)} F$ are out-of-the-model derivatives while the one in the proof on options on futures are in-the-model rate movements.

Proof (Using model structure). The discount factor ratios are

$$
\frac{P\left(t, t_{i, j}\right)}{P\left(t, t_{0}\right)}=\frac{P\left(0, t_{i, j}\right)}{P\left(0, t_{0}\right)} \beta_{i, j}(0, t) \exp \left(-\frac{1}{2} \alpha_{i, j}^{2}(0, t)-\alpha_{i, j}(0, t) X_{t}\right)
$$

with $X_{t}$ a $\mathbb{N}$-normally distributed random variable.

For each value of $X$, the ratios are different. The sequences $\kappa_{i}$ and $m_{i}$ associated are also potentially different.

If the $m_{i}$ and $k$ of (Henrard, 2006a, Theorem 2) were actually different it would complicate substantially the task. One would have a number of terms in the sum that represents the value of the futures $F_{t}$ which is stochastic.

With a technique similar to the one used in (Henrard, 2006b, Theorem 4) it can be proved that the number of terms $k$ and their order $m_{i}$ is constant for all value of $X$. The constance is proved by showing that the number and order of intersection points between all the $f_{i}$ curves pairs are always the same.

The intersection between two curves is at point $\kappa_{p, q}\left(X_{t}\right)$ that satisfies

$$
\sum_{j=0}^{n_{p, q}} d_{p, q, j}(t, \theta) \frac{P\left(0, t_{p, q, j}\right)}{P\left(0, t_{0}\right)} \exp \left(-\frac{1}{2} \alpha_{p, q, j}^{2}(0, \theta)-\alpha_{p, q, j}(0, t) X_{t}-\alpha_{p, q, j}(t, \theta) \kappa\right)=0 .
$$

The standard deviation coefficients can be written using $(\mathrm{H})$ as

$$
\alpha_{p q, j}^{2}(u, v)=H^{2}\left(t_{p q, j}\right)(G(v)-G(u))
$$

and

$$
\begin{gathered}
\alpha_{i, j}(0, t) X_{t}=\int_{0}^{t} \nu\left(s, t_{i, j}\right)-\nu\left(s, t_{0}\right) d W_{s} \\
\alpha_{i, j}(0, t) d X_{t}=\ldots+\left(\nu\left(t, t_{i, j}\right)-\nu\left(t, t_{0}\right)\right) d W_{t} .
\end{gathered}
$$

Let $\Lambda$ be the $X_{t}$-independent solution(s) of (Henrard, 2006a, Equation 3).

The solution $\kappa_{p q}\left(X_{t}\right)$ of the initial equation can be written through

$$
\kappa_{p q}\left(X_{t}\right)=\kappa\left(\Lambda_{p q}, X_{t}\right)
$$

with

$$
\kappa(\Lambda, X)=\frac{\Lambda-\sqrt{-G(0)} X}{\sqrt{G(\theta)}} .
$$


M. HENRARD

All the $\kappa_{p, q}(1 \leq p, q \leq k)$ have the same order as the $\Lambda_{p, q}$. This was proved in Theorem 4 in Henrard (2006a).

With all these ingredients in place the futures price can be written as

$$
\begin{gathered}
F_{t}=\sum_{i=1}^{k} \sum_{j=0}^{n_{m_{i}}} d_{m_{i}, j}(t, \theta) \frac{P\left(0, t_{i, j}\right)}{P\left(0, t_{0}\right)} \beta_{m_{i}, j} \exp \left(-\frac{1}{2} \alpha_{m_{i}, j}^{2}(0, t)-\alpha_{m_{i}, j}(0, t) X_{t}\right) \\
\left(N\left(\kappa\left(\Lambda_{i}, X_{t}\right)+\alpha_{m_{i}, j}(t, \theta)\right)-N\left(\kappa\left(\Lambda_{i-1}, X\right)+\alpha_{m_{i}, j}(t, \theta)\right)\right) .
\end{gathered}
$$

The variation of $F_{t}$ is then

$$
\begin{aligned}
d F_{t}= & \cdots-\sum_{i=1}^{k} \sum_{j=0}^{n_{m_{i}}} d_{m_{i}, j}(t, \theta) \frac{P\left(0, t_{m_{i}, j}\right)}{P\left(0, t_{0}\right)} \beta_{m_{i}, j} \exp \left(-\frac{1}{2} \alpha_{m_{i}, j}^{2}(0, t)-\alpha_{m_{i}, j}(0, t) X_{t}\right) \\
& \left(N\left(\kappa\left(\Lambda_{i}, X_{t}\right)+\alpha_{m_{i}, j}(t, \theta)\right)-N\left(\kappa\left(\Lambda_{i-1}, X\right)+\alpha_{m_{i}, j}(t, \theta)\right)\right) \alpha_{m_{i}, j}(0, t) d X_{t} \\
+ & \sum_{i=1}^{k-1} \sum_{j=0}^{n_{m_{i}}} d_{m_{i}, j}(t, \theta) \frac{P\left(0, t_{m_{i}, j}\right)}{P\left(0, t_{0}\right)} \beta_{m_{i}, j} \exp \left(-\frac{1}{2} \alpha_{m_{i}, j}^{2}(0, t)-\alpha_{m_{i}, j}(0, t) X_{t}\right) \\
& N^{\prime}\left(\kappa\left(\Lambda_{i}, X_{t}\right)+\alpha_{m_{i}, j}(t, \theta)\right) D_{2} \kappa\left(\Lambda_{i}, X_{t}\right) d X_{t} \\
& \sum_{i=1}^{k-1} \sum_{j=0}^{n_{m_{i+1}}} d_{m_{i+1}, j}(t, \theta) \frac{P\left(0, t_{m_{i+1}, j}\right)}{P\left(0, t_{0}\right)} \beta_{m_{i+1}, j} \exp \left(-\frac{1}{2} \alpha_{m_{i+1}, j}^{2}(0, t)-\alpha_{m_{i+1}, j}(0, t) X_{t}\right) \\
& N^{\prime}\left(\kappa\left(\Lambda_{i}, X_{t}\right)+\alpha_{m_{i+1}, j}(t, \theta)\right) D_{2} \kappa\left(\Lambda_{i}, X_{t}\right) d X_{t} .
\end{aligned}
$$

The dots indicate the constant and $d t$ terms. As the future price is a martingale, the $d t$ terms are nuls. Using the equations caracterising $\kappa_{i}$, the two last term of the equation are nul.

Rearranging the terms and using the caracterisitic of $\alpha d X_{t}$ given above lead to the result.

\section{A singular example}

An example where the non-degenaracy condition is not satisfied is provided. It is also proved that for the same example the second order partial derivative (gamma) does not exists.

The example is similar to the third example in Henrard (2006a). There are two bonds with one cash-flow each. The parameters are given in Table 1.

\begin{tabular}{lllll}
\hline \multicolumn{5}{c}{ Singular cross } \\
\hline$i$ & $j$ & $e_{i}$ & $d_{i, j}$ & $\alpha_{i, j}$ \\
1 & 1 & 0 & 1 & 1 \\
2 & 1 & $y_{0}$ & 3 & $1 / 3$ \\
\hline \multicolumn{4}{l}{ Table Note } \\
\hline
\end{tabular}

TABLE 1. Constant for two bonds with one singular intersection

The initial discount factors are chosen to be 1 . The derivative to the discount factor associated to the maturity in the second bond is analysed. The constant $y_{0}$ is chosen in such a way that there is one degenerate cross-over point. It's value is $2 * \exp (1 / 6)$. With those constants one has

$$
\begin{aligned}
& f_{1}(P, x)=\exp (-1 / 2-x) \\
& f_{2}(P, x)=3 P \exp (-1 / 18-1 / 3 x)-y_{0} .
\end{aligned}
$$

The two functions are equal in $x=-2 / 3$. This is the only intersection point and for the other points $f_{1}(1, x)>f_{2}(1, x)$. 
It can be shown that for $P<1$ there is no intersection and for $P>1$ there are two intersections. The value of the futures is

$$
\begin{array}{cc}
P \leq 1 & F_{0}(P)=3 P-y_{0} \\
P>1 & \bar{F}_{0}(P)=3 P N\left(\kappa_{1}+\alpha_{2}\right)-y_{0} N\left(\kappa_{1}\right)+\left(N\left(\kappa_{2}+\alpha_{1}\right)-N\left(\kappa_{1}-\alpha_{1}\right)\right) \\
& +3 P\left(\left(1-N\left(\kappa_{2}+\alpha_{2}\right)\right)-y_{0}\left(1-N\left(\kappa_{2}\right)\right) .\right.
\end{array}
$$

The function is continuous and $\lim _{P \rightarrow<1} F_{0}(P)=\lim _{P \rightarrow>1} \bar{F}_{0}(P)=3-y_{0}$.

The derivative of the two parts of the function can be computed. Using the details in the proof,

$$
\begin{array}{ll}
P \leq 1 & F_{0}^{\prime}(P)=3 \\
P>1 & \bar{F}_{0}^{\prime}(P)=3 N\left(\kappa_{1}+\alpha_{2}\right)+3\left(1-N\left(\kappa_{2}+\alpha_{2}\right)\right) .
\end{array}
$$

Here also the left and right limits are equal with $\lim _{P \rightarrow<1} F_{0}^{\prime}(P)=3=\lim _{P \rightarrow>1} \bar{F}_{0}^{\prime}(P)$. This proves that the futures price is $C^{1}$ in $P$ around $P=1$.

Nevertheless this can not be deduced directly from the form of $F_{0}^{\prime}$ as the function $\kappa_{i}$ are not well defined for $P \leq 1$. For $P>1$ they exists but their derivative tend to infinity for $P \rightarrow 1$. The graph of the $\kappa$ 's is displayed in Figure 1(a).

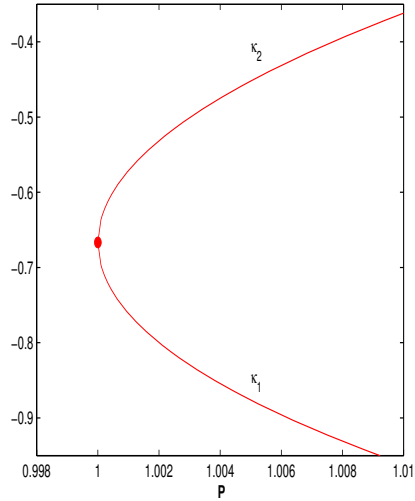

(a) $\kappa$

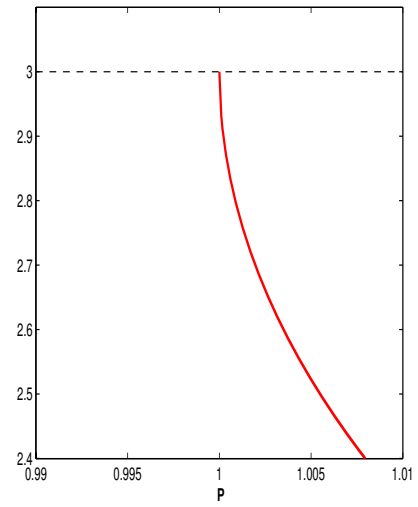

(b) Delta

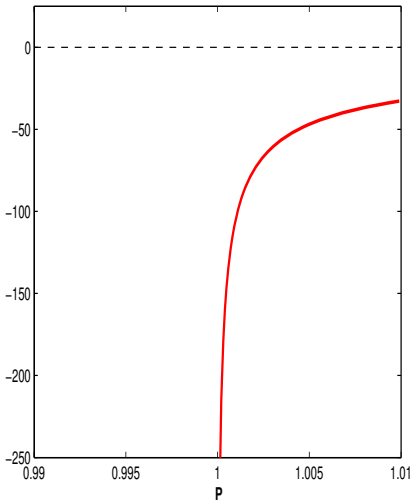

(c) Gamma

Figure 1. Price dependence of $\kappa$, delta and gamma.

The second derivatives are given by

$$
\begin{array}{ll}
P \leq 1 & F_{0}^{\prime \prime}(P)=0 \\
P>1 & \left.\bar{F}_{0}^{\prime \prime}(P)=3 N^{\prime}\left(\kappa_{1}(P)+\alpha_{2}\right) \kappa_{1}^{\prime}(P)-3 N^{\prime}\left(\kappa_{2}(P)+\alpha_{2}\right)\right) \kappa_{2}^{\prime}(P) .
\end{array}
$$

But $\kappa_{1}^{\prime}(P) \rightarrow-\infty$ and $\kappa_{3}^{\prime}(P) \rightarrow+\infty$ as $P \rightarrow>1$ and $N^{\prime}$ is positive. So $F_{0}^{\prime \prime}$ tends to $-\infty$ as $P \rightarrow>1$. The graphs of the first and second order derivatives are given in Figure 1(b) and 1(c).

The example proves that the second order partial derivatives does not always exist. Several questions are still open. The first one is the existence of the first order derivatives. It was proved that for non degenerate $\kappa$ they exist. From the example there exists cases where they exist even if the crossing is degenerate and the $\kappa$ 's are non differentiable. The general proof of the existence or the description of the conditions of existence is still an open problem. For the second order derivatives, the existence is proved for non-degenerate crossing points and an example of nonexistence is given. The precise conditions of non-existence are still open. 


\section{Conclusion}

The note discuss several rate risk measures for bond futures: in-the-model delta and out-of-themodel delta and gamma.

A general formula for the in-the-model delta is provided. The best proof of the general result does not use standard approach through partial derivatives. The stochastic differential with respect to the factor of the model is used instead. The proof is less standard but is robust and uses more the structure of the model.

The out-of-the-model delta and gamma or first and second order partial derivatives with respect to the zero-coupon prices are studied. Under a non-degeneracy condition of the crossing points those derivatives exists. A relatively simple example proves that in somes cases second order derivatives does not exists. Between those two results several existence questions in the degenerate case are still open.

Disclaimer: The views expressed here are those of the author and not necessarily those of the Bank for International Settlements.

\section{REFERENCES}

Brody, D. C. and Hughston, L. P. (2004). Chaos and coherence: a new framework for interest-rate modelling. Proc. R. Soc. Lond. A., 460:85-110.

Carverhill, A. (1994). When is the short rate Markovian. Mathematical Finance, 4(4):305-312.

Heath, D., Jarrow, R., and Morton, A. (1992). Bond pricing and the term structure of interest rates: a new methodology for contingent claims valuation. Econometrica, 60(1):77-105.

Henrard, M. (2003). Explicit bond option and swaption formula in Heath-Jarrow-Morton one-factor model. International Journal of Theoretical and Applied Finance, 6(1):57-72.

Henrard, M. (2006a). Bonds futures and their options: more than the cheapest-to-deliver; quality option and marginning. Technical report, SSRN.

Henrard, M. (2006b). A semi-explicit approach to Canary swaptions in HJM one-factor model. Applied Mathematical Finance, 13(1):1-18.

Hunt, P. J. and Kennedy, J. E. (2004). Financial Derivatives in Theory and Practice. Wiley series in probability and statistics. Wiley, second edition.

Lamberton, D. and Lapeyre, B. (2000). Introduction to stochastic calculus applied to finance. Capman \& Hall / CRC.

Nunes, J. and de Oliveira, L. (2004). Quasi-analytical multi-factor valuation of treasury bond futures with and embedded quality option. Technical Report 2493, EFA 2004 Maastricht Meeting.

Derivatives Group, Banking Department, Bank for International Settlements, CH-4002 Basel (SwitzerLAND)

E-mail address: Marc.Henrard@bis.org 